\title{
Julia Anna Riedel, Bildungsreform und geistliches Ordenswesen im Ungarn der Aufklärung. Die Schulen der Piaristen unter Maria Theresia und Joseph II
} Stuttgart : Steiner (Contubernium. Tübinger Beiträge zur Universitätsund Wissenschaftsgeschichte, 77), 2012, XIV-611 p., $87 €$

Juliette Guilbaud

\section{OpenEdition}

\section{Journals}

Édition électronique

URL : http://journals.openedition.org/ifha/8152

DOI : $10.4000 /$ ifha. 8152

ISSN : 2198-8943

Éditeur

IFRA - Institut franco-allemand (sciences historiques et sociales)

Référence électronique

Juliette Guilbaud, « Julia Anna Riedel, Bildungsreform und geistliches Ordenswesen im Ungarn der Aufklärung. Die Schulen der Piaristen unter Maria Theresia und Joseph II », Revue de I'IFHA [En ligne], Date de recension, mis en ligne le 14 avril 2015, consulté le 22 septembre 2020. URL : http:// journals.openedition.org/ifha/8152 ; DOI : https://doi.org/10.4000/ifha.8152

Ce document a été généré automatiquement le 22 septembre 2020.

CIFHA 


\section{Julia Anna Riedel, Bildungsreform und geistliches Ordenswesen im Ungarn der Aufklärung. Die Schulen der Piaristen unter Maria Theresia und Joseph II}

Stuttgart : Steiner (Contubernium. Tübinger Beiträge zur Universitätsund Wissenschaftsgeschichte, 77), 2012, XIV-611 p., $87 €$

Juliette Guilbaud

Ce copieux volume, issu d'une thèse d'histoire soutenue par l'auteure en 2010 à l'université de Tübingen, traite de la réforme de l'enseignement dans la Hongrie du $\mathrm{XVIII}^{\mathrm{e}}$ siècle. Cette réforme est l'occasion d'une confrontation entre le pouvoir monarchique - sous les règnes de Marie-Thérèse puis de Joseph II (précisément entre 1773 et 1790 - et l'un des principaux ordres enseignants alors présents dans le royaume, celui des piaristes. Ces deux acteurs se révèlent portés par des intérêts différents - tantôt convergents, tantôt contradictoires - que Julia Anna Riedel s'emploie à analyser finement dans son ouvrage.

Outre le trio classique problématique - méthodologie - état de la recherche, ainsi qu'une rapide présentation des sources, l'introduction explicite utilement les notions clés sur lesquelles l'auteure appuie sa démonstration et dont d'ailleurs, pour certaines, la traduction vers le français n'est pas sans poser régulièrement problème: Orden/ Ordensgeistlicher (ordre religieux/religieux vs. régulier), Schule, Säkularisierung, katholische Aufklärung, Josephinismus, aufgeklärter Absolutismus. Le développement s'articule ensuite autour de deux grandes parties, la première sur les piaristes et le système éducatif dans le royaume de Hongrie ; la seconde, plus fournie, sur l'ordre, sa participation à la réforme de l'éducation et sa capacité à se réformer soi-même.

Les piaristes, relativement moins connus en France que d'autres réguliers, sont fondés au début du XVII e siècle par l'Espagnol Joseph Calasanz (1556-1648); élevés au rang d'ordre religieux en 1621 par Grégoire XV, ils essaiment en particulier en Italie et dans l'Europe centrale moderne. Ils comptent ainsi parmi les réguliers les plus répandus 
dans la monarchie Habsbourg au XVIII ${ }^{\mathrm{e}}$ siècle. En Hongrie notamment, ils sont des acteurs majeurs de l'enseignement primaire et secondaire. Dans le secondaire en particulier, ils entrent en concurrence avec les jésuites, avant la suppression de la Compagnie de Jésus en 1773. Dans cette première partie également, Julia Anna Riedel se penche sur le rapport des piaristes à l'Aufklärung, à travers le portrait de plusieurs membres de l'ordre impliqués par leurs écrits dans les débats du temps. Est ainsi mise en évidence une évolution, celle du religieux enseignant vers la figure du clerc savant, ouvert sur le siècle et faisant œuvre littéraire (der geistliche bürgerliche Gelehrte). Si l'on peut citer quelques tenants des Lumières radicales au sein de l'ordre, les piaristes apparaissent majoritairement ouverts à une Aufklärung modérée.

Dans la seconde grande partie, l'auteure s'interroge sur le sens de la réforme d'un système éducatif largement dominé par l'Église à travers ses ordres enseignants dont les piaristes, en Hongrie, sont en quelque sorte les têtes de pont. Dans quelle mesure la réforme peut-elle être comprise comme une arme dirigée par le pouvoir contre l'ordre ? Et comment fut-elle perçue par les piaristes eux-mêmes ? C'est à ces questions que tente de répondre notamment l'explicite chapitre "Coopération et confrontation » (p. 258-287). Plus qu'un bouleversement des contenus et malgré l'introduction de nouvelles matières, la réforme révèle la volonté des souverains de placer l'enseignement sous le contrôle de l'État (c'est en ce sens qu'il faut comprendre la " sécularisation »), sans pour autant renoncer à un ensemble de pratiques qui ont fait leurs preuves, notamment sous l'égide des piaristes. On rappellera par exemple la permanence et l'importance en Hongrie de l'enseignement du latin qui reste longtemps la langue de l'administration (officiellement jusqu'en 1844). Les piaristes apparaissent donc au départ comme des partenaires ouverts à la réforme de l'éducation (Ratio educationis de 1777), car elle ne remet pas fondamentalement en cause leurs méthodes. Mais les difficultés se font jour en raison des exigences des autorités vis-à-vis de l'ordre pour la mise en œuvre de cette réforme. Ces résistances se manifestent plutôt sous Joseph II, par l'intermédiaire du provincial piariste. La correspondance de ce dernier en particulier révèle les difficultés financières de l'ordre, mais aussi les déficits de formation et de recrutement de ses membres, toutes choses qui empêcheraient les piaristes de satisfaire les exigences de l'État. Seule l'initiative de l'ordre de participer à sa propre réforme après 1790 , en collaboration avec le pouvoir monarchique, lui permet de tirer son épingle du jeu après avoir échappé à la suppression massive des ordres religieux en 1782 : les piaristes se révèlent indispensables dans l'enseignement en Hongrie et arguent de ces fonctions - ils œuvrent en quelque sorte pour le bien commun - pour justifier leur maintien à la fin du XVIII ${ }^{e}$ siècle.

Si l'approche privilégiée dans cet ouvrage n'est pas des plus originales, on ne peut que saluer le sérieux avec lequel est analysé un corpus de sources multilingue (allemand, hongrois, latin) et de provenance croisée (sources de l'ordre/sources de l'administration « séculière »), qui permet à l'auteure d'analyser son objet avec la plus grande précision et nuance. On saluera enfin l'abondance et la diversité des annexes (cartes, graphiques, tableaux et près de 40 illustrations), ainsi que la présence d'un index multilingue des plus roboratifs dont la réalisation, pour établir les concordances entre la presque dizaine d'idiomes de la région, représente en soi un véritable tour de force. 
INDEX

Index chronologique : Période moderne

Thèmes : Histoire religieuse, Histoire de la culture, Histoire des idées

\section{AUTEUR}

JULIETTE GUILBAUD

CNRS, centre Georg-Simmel (UMR 8131) 